\title{
Evaluation of Clinical Symptoms Related to Common Serogroups of Leptospirosis in North of Iran
}

\author{
Mostafa Javanian ${ }^{1}$, Mahmoud Sadeghi Haddad Zavareh ${ }^{1}$, Nasrollah Vahedi ${ }^{2}$, Arefeh Babazadeh ${ }^{1} \&$ \\ Soheil Ebrahimpour ${ }^{1}$ \\ ${ }^{1}$ Infectious Diseases and Tropical Medicine Research Center, Health Research Institute, Babol University of \\ Medical Sciences, Babol, I.R. Iran \\ ${ }^{2}$ Member of Scientific Board in Research Center of Agriculture, Mazandaran, Iran \\ Correspondence: Mahmoud Sadeghi Haddad Zavareh, Infectious Diseases and Tropical Medicine Research Center, \\ Health Research Institute, Babol University of Medical Sciences, Babol, I.R. Iran. Tel: 98-911-311-5047. Email: \\ drm-sadeghihz@yahoo.com
}

Received: September 2, 2017

Accepted: September 12, 2017 Online Published: September 18, 2017

doi:10.5539/jmbr.v7n1p127

URL: https://doi.org/10.5539/jmbr.v7n1p127

\begin{abstract}
Leptospirosis is a common disease between humans and animals. Identifying common serogroups and comparing different clinical symptoms among them can help in finding the clinical pattern associated with pathogen serogroupes of leptospirosis. Therefore, the purpose of this study has been to investigate the common serotypes of the infection and their clinical symptoms in northern Iran. This cross-sectional study was carried out in educational hospitals of Babol University of Medical Sciences during the years 2011- 2014. Subjects with clinical findings consistent with leptospirosis were included in the study. According to the standard MAT guidelines, the titre $\geq 1$ : 200 was considered positive. Then, the patients identified by serogroup separation, were examined and compared clinical symptoms. Among 6o patients with primary diagnosis of leptospirosis in this study, 35 of them proved to be infected to the disease. The most common serogroups were serjoe $(40 \%)$ and icterohemorrhagia $(31.4 \%)$. Autumnalis (22.8\%), grippotyphosa (11.4\%), canicola (8.6\%), and pomona (2.8\%) were included the subsequent serogroups. The highest frequency of fever (28\%) and gastrointestinal manifestations $(36 \%)$ were observed in icterohemorrhagia and the highest icterus $(30 \%)$ was found in serjoe serogroup. In this study, serjoe serogroup with fever and icterus, and then icterohemorrhagia with fever and gastrointestinal symptoms were introduced as the most common serogroups of Leptospirosis. Also the rarest serogroups were canicola and pomona.
\end{abstract}

Keywords: Leptospirosis, Serogroup, Clinical symptoms, North of Iran

\section{Introduction}

Leptospirosis is a common zoonotic infectious diseases, caused by spirochetes from the leptospirosis family and preserved by chronic renal infection of carrier animals, especially rodents in nature (Barragan et al., 2017). Leptospirosis is more prevalent in tropical and temperate regions, especially in the sultry ones, and is endemic in most of these areas (Joshi et al., 2017; Palihawadana et al., 2014). This infection is transferred to humans through occupational or recreational exposure to water or soil contaminated with the infected rodents' urine. The infection in developing countries such as Iran is considered as an occupational disease, and is mainly seen among farmers and ranchers in the warm seasons ( Benacer et al., 2016; Ghasemian et al., 2016). Today, leptospirosis is proposed as a major public sanitation problem in developing countries due to epidemic and increasing prevalence. Leptospirosis infection is associated with a wide range of clinical symptoms as a subclinical infection, self-limiting systemic disease in $90 \%$ of cases and or severe fatal disease with multiple-organ failure (Koe et al., 2014). Classically, this is a two-phase disease that, after 10-day latency, acute septicemic phase begins with sudden fever, chilling, headache, myalgia, and gastrointestinal symptoms. This phase lasts about a week. After that, the immune phase, which lasts for about 4 days to one month, begins ( Daher et al., 2010). An aseptic meningitis that can be asymptomatic is a characteristic of this phase of infection. Leptospira genus includes 21 species that is divided into 2 general groups of leptospira intrrogans and leptospira biflexa, which include pathogenic and non-pathogenic genomes. There are a large number of serogroups in each gene. So far, over 250 Leptospira pathogen serogroups have been described (Haake \& Levett, 2015; Lehmann et al., 2014; Mehrotra et al., 2017). 
The north of the country, especially mazandaran province, due to its temperate and humid climate, high annual rainfall, the presence of rice paddies, abundance of wild and domestic animals, creates favorable conditions for the development of Leptospirosis infection agent, and a large number of patients suspected of being infected with this infection being hospitalized yearly, which the definitive diagnosis and determination of the serotype causing the disease in these patients is usually remains unknown. It should be noted that the determination of common and endemic serovars in each area is very important and is considered the first step in epidemiological studies. As we know, the symptoms of leptospirosis include a wide range of mild disease with the flu-like manifestations to severe form with kidney and liver involvement and bleeding (Haake \& Levett, 2015). Therefore, studying different serogroups of leptospirosis agent in the region and comparing different clinical symptoms among them can be helpful in finding the clinical pattern of serovars of leptospirosis pathogen and also, can manifest the cause of the difference in the manifestations of this infection among different regions. Therefore, in the present study, we investigated the common serogroups of leptospirosis and their related clinical symptoms in north of Iran.

\section{Materials and Methods}

In this cross-sectional study conducted in Babol University of Medical Sciences during the academic years 20112014, people who, according to the World Health Organization, have clinical findings consistent with leptospirosis infection such as fever, headache, icterus, myalgia, anorexia, nausea, and vomiting and also a similar epidemiologic history, such as exposure with contaminated water with animal sources or agricultural activities were included in the study (Organization, 2010). Children under the age of 8, people with immunodeficiency and chronic diseases were excluded from the study. A questionnaire containing demographic information, clinical and laboratory symptoms was completed for all patients. Of these subjects, $5 \mathrm{ml}$ blood sample was taken to carry out the MAT serologic test. According to the standard guidelines, the titre $\geq 1: 200$ was considered positive (Yitzhaki et al., 2004). In this study, MAT panel of 23 serovars was used. Then, the patients were identified by serogroup separation, examined and compared for clinical manifestations. The protocol was approved by the Ethical Committee of Babol University of Medical Sciences. Informed consent was completed by all patients participating in this research project. It is worth noting that all the results were analyzed by SPSS16 software and $\mathrm{p} \leq 0.05$ was considered significant.

\section{Results}

In the present study, 60 patients with primary diagnosis of leptospirosis based on the criteria presented in valid scientific literature were serologically tested using the standard MAT method that, in 35 people the diagnosis was confirmed. Of the patients with the diagnosis of infection, 31 (88.6\%) were males and $30(85 \%)$ were farmers (Table1). The most common clinical symptom of these patients (91.4\%) was fever, followed by chilling (48.6\%), myalgia (40\%), and icterus (37.2\%). At the next level, gastrointestinal complaints (31\%) and headache (22.9\%) were the rarest symptoms of the disease. The most common serogroupes in the present study were in titers between 1: 200 to 1: 12800 , serjoe (40\%), and ictrohemorrhagia (31.4\%). Autumnalis (22.8\%), grippotyphosa (11.4\%), canicola $(8.6 \%)$, and pomona $(2.8 \%)$ formed the next serogroups. It is worth noting that some patients were simultaneously infected with several serogroups. The highest frequency of fever $(28 \%)$ and gastrointestinal manifestations (36\%) was found in icterohemorrhagia serogroup and the most icterus (30\%) was observed in serjoe serogroup. The highest headache frequency $(37.5 \%)$ was observed in people who were simultaneously infected with serjoe and hardjo serotypes. In the patients infected with serjoe serogroup, the most common symptom was fever $(80 \%)$ and then icterus $(60 \%)$. In icterohemorrhagia serogroup, the most common symptoms were fever $(100 \%)$, chilling $(44.4 \%)$, gastrointestinal symptoms $(44 \%)$ and headache $(37.5 \%)$. The rarest symptoms were icterus (22\%) and myalgia (22.2\%). The most observed symptoms in patients with autumnalis serogroup were fever and chilling $(100 \%)$ and myalgia $(60 \%)$. In the present study, all cases contaminated with hardjo were observed with other serogroups (Table2 and 3). So the study of the symptoms associated with this serogroup as a common serogroup was not possible independently.

Table 1. Demographical data in the patients with leptospirosis

\begin{tabular}{lll}
\hline \multicolumn{2}{l}{ Age(years), Mean \pm SD } & $\mathbf{4 9 . 2 8} \pm \mathbf{9 . 2 5}$ \\
\hline Sex & Male, $\mathbf{n}(\%)$ & $31(88.6 \%)$ \\
& Female, $\mathbf{n}(\%)$ & $4(11.4 \%)$ \\
\multirow{2}{*}{ Occupation } & Farmer, n (\%) & $30(85.7 \%)$ \\
& Others, $\mathbf{n}(\%)$ & $5(14.3 \%)$ \\
\hline
\end{tabular}


Table 2. Clinical finding of serogroups

\begin{tabular}{|c|c|c|c|c|c|c|c|c|c|c|c|}
\hline & \multicolumn{10}{|c|}{ Serogroups } & \multirow{2}{*}{ Total } \\
\hline & 1 & 2 & 3 & 4 & 5 & 6 & 7 & 8 & 9 & 10 & \\
\hline Fever & $4(12.5 \%)$ & $9(28.1 \%)$ & $2(6.3 \%)$ & $1(3.1 \%)$ & $5(15.6 \%)$ & $7(21.9 \%)$ & $1(3.1 \%)$ & $1(3.1 \%)$ & $1(3.1 \%)$ & $1(3.1 \%)$ & $32(91.4 \%)$ \\
\hline Chilling & $2(11.8 \%)$ & $4(23.5 \%)$ & $2(11.8 \%)$ & $1(5.9 \%)$ & $5(29.4 \%)$ & $2(11.8 \%)$ & 0 & 0 & 0 & $1(5.9 \%)$ & $17(48.6 \%)$ \\
\hline Sweating & 0 & $2(50 \%)$ & 0 & 0 & $1(25 \%)$ & 0 & 0 & 0 & $1(25 \%)$ & 0 & $4(11.4 \%)$ \\
\hline Ichter+ Pruritus & $4(30 \%)$ & $2(15 \%)$ & 0 & $2(15 \%)$ & $2(15 \%)$ & $1(7 \%)$ & 0 & $1(7 \%)$ & 0 & $1(7 \%)$ & $13(37.2 \%)$ \\
\hline Headache & 0 & $3(37.5 \%)$ & 0 & 0 & $2(25 \%)$ & $3(37.5 \%)$ & 0 & 0 & 0 & 0 & $8(22.9 \%)$ \\
\hline GI complaint & $2(18 \%)$ & $4(36 \%)$ & $1(9 \%)$ & 0 & 0 & $2(18 \%)$ & $2(18 \%)$ & 0 & 0 & 0 & $11(31 \%)$ \\
\hline Myalgia & $1(7.1 \%)$ & $2(14.3 \%)$ & $1(7.1 \%)$ & 0 & $3(21.4 \%)$ & $5(35.7 \%)$ & 0 & $1(7.1 \%)$ & $1(7.1 \%)$ & 0 & $14(40 \%)$ \\
\hline
\end{tabular}

1: Serjoe, 2: Icterohaemorrhagiae, 3: Grippotyphosa, 4: Canicola, 5: Atumnalis, 6: Serjoe+ Hardjo, 7: Icterohaemorrhagiae + Canicola, 8: Icterohaemorrhagiae+Pomona, 9: Serjoe + Grippotyphosa + Hardjo + Atumnalis, 10: Serjoe+ Grippotyphosa + Icterohaemorrhagiae + Atumnalis + Pomona

Table 3. Frequency of leptospirosis patients with more than one serogroup

\begin{tabular}{|c|c|c|c|c|c|c|c|}
\hline Frequency $(\%)$ & Serjoe & Icterohemorrhagia & Grippotyphosa & Hardjo & Autumnalis & canicola & Pomona \\
\hline $20 \%$ & $*$ & & & $*$ & & & \\
\hline $2.9 \%$ & & $*$ & & & & & $*$ \\
\hline $2.9 \%$ & & $*$ & & & & $*$ & \\
\hline $2.9 \%$ & $*$ & & $*$ & $*$ & $*$ & & \\
\hline $2.9 \%$ & $*$ & $*$ & & $*$ & & & \\
\hline
\end{tabular}

\section{Discussion}

In this study, the most common symptoms were fever (91.4\%), chilling (48.6\%) and myalgia (40\%). Gastrointestinal manifestations (31\%) and headache (22.9\%) were the next most frequent ones. In a study carried out in Sari, Aaliyan et al. reported that fever (96.6\%), headache (93.1\%), and then myalgia (85\%) and chilling (86.2\%) were the most common symptoms (Alian et al., 2015). Honarmand et al., during the study of a decade of leptospirosis incidence in Guilan, reported that fever, myalgia, icterus and conjunctivitis were the most common clinical symptoms (Honarmand et al., 2007). In the study of Golsha et al. in Golestan, fever, chilling and myalgia $(100 \%)$ were reported as the most common clinical symptoms of leptospirosis (Golsha et al., 2007). Fever and myalgia were the most common symptoms of leptospirosis in Zakeri et al.'s research (Zakeri et al., 2010). In the study of Sehgal and Katz, among the various clinical symptoms, fever and headache have been referred as the most common symptoms (Katz et al., 2011; Sehgal, 2006). In the present study, myalgia is more rarely complaint than other studies, which may be due to excessive consumption of lenitive and also, due to the possible association between the type of serogroup and the manifestations of the disease; the difference between the common serogroups of the disease in the north of Iran should be more carefully considered than other regions. In our study, serjoe was the most common serogroup and after that the icterohemorrhagia was in the second place. However, in Ghenaeei et al.'s study, icterohemorrhagia was the most common serogroup and any case of serjoe was not reported (Mansour-Ghanaei et al., 2005). In the study of Tasseva et al. in Bulgaria, iterohemorrhagia and pomona serogroups were the most common causes of the disease (Tasseva et al., 2012). In another study that performed in Guilan, the species of interrogans was the most common gene (Honarmand et al., 2005). In a study carried out by Faraji et al. in north of Iran, baloum, serjoe and australis had been reported as the most common serogroups (Faraji et al., 2016). In a study in Denmark, like our study, icterohemorrhagia and serjoe, were the most common serogroups (Holk et al., 2000). It seems that clinical symptoms associated with different serogroups can reveal the difference between the clinical symptoms of the disease in different parts of the world, and so far this has not been fully done in other studies. In this study, we investigated the clinical symptoms of different serogroups of this disease agent. In serjoe serogroup, fever $(80 \%)$ and icterus $(60 \%)$ have been the most common clinical symptoms. The most common symptoms, in icterohemorrhagia serogroup, were fever $(100 \%)$ and gastrointestinal symptoms (44.4\%), and in the case of autumnalis, fever and chilling (100\%) and then myalgia (60\%) were the most common complaints of the patients. regarding that comparing the clinical symptoms between different serogroups can reveal the characteristics related with each serogroup and justify the common clinical picture of the disease in the region, it is recommended that the studies with a larger sample size be designed to study this important case. 


\section{Conclusions}

In this study, male farmers had the highest incidence number. The most common serogroups of the disease agent was serjoe with fever and icterus, and next was icterohemorrhagia with fever and gastrointestinal symptoms. Also the rarest serogroups were canicola and pomona.

\section{Acknowledgments}

Hereby, this is appreciated the deputy of research and technology of Babol University of Medical Sciences for supporting of this research and the staffs of Ayatollah Rouhani and Research Center of Agriculture, Mazandaran for their cooperation.

\section{References}

Alian, S., Davoudi, A., Najafi, N., Ghasemian, R., Ahangarkani, F., \& Hamdi, Z. (2015). Clinical and laboratory manifestation and outcome of icterohemorrhagic leptospirosis patients in Northern Iran. Medical Journal of the Islamic Republic of Iran, 29, 308-308.

Barragan, V., Nieto, N., Keim, P., \& Pearson, T. (2017). Meta-analysis to estimate the load of Leptospira excreted in urine: beyond rats as important sources of transmission in low-income rural communities. BMC research notes, 10(1), 71. https://doi.org/10.1186/s13104-017-2384-4

Benacer, D., Thong, K. L., Verasahib, K. B., Galloway, R. L., Hartskeerl, R. A., Lewis, J. W., \& Mohd Zain, S. N. (2016). Human leptospirosis in Malaysia: reviewing the challenges after 8 decades (1925-2012). Asia Pacific Journal of Public Health, 28(4), 290-302. https://doi.org/10.1177/1010539516640350

Daher, E. F., Lima, R. S., Silva Júnior, G. B., Silva, E. C., Karbage, N. N., Kataoka, R. S., . . Libório, A. B. (2010). Clinical presentation of leptospirosis: a retrospective study of 201 patients in a metropolitan city of Brazil. Brazilian Journal of Infectious Diseases, 14(1), 03-10.

Faraji, H., Mirzaei, H., Afshar, D., Nouri, P., Roshanjo, K., Mohamadi Bardebari, A., \& Mirzaei, H. R. (2016). The Epidemiology of Leptospira Infection in Mazandaran Province, Northern Iran, During 2012 - 2013. Iranian Red Crescent Medical Journal, 18(7), e23194. https://doi.org/10.5812/ircmj.23194

Ghasemian, R., Shokri, M., Makhlough, A., \& Suraki-Azad, M. A. (2016). The course and outcome of renal failure due to human leptospirosis referred to a hospital in North of Iran; A follow-up study. Caspian journal of internal medicine, 7(1), 7.

Golsha, R., Khodabakhshi, B., \& Rahnama, A. (2007). Leptospirosis in Golestan province in Iran (Reports of twelve cases).

Haake, D. A., \& Levett, P. N. (2015). Leptospirosis in Humans. Current topics in microbiology and immunology, 387, 65-97. https://doi.org/10.1007/978-3-662-45059-8_5

Holk, K., Nielsen, S. V., \& Rønne, T. (2000). Human leptospirosis in Denmark 1970-1996: an epidemiological and clinical study. Scandinavian journal of infectious diseases, 32(5), 533-538.

Honarmand, H., Eshraghi, S., Khorramizadeh, M., Hartskeerl, R., Ghanaei, F., Abdolahpour, G., \& Eshraghian, M. (2007). Distribution of.Human Leptospirosis in Guilan Province, Northern Iran. Iranian Journal of Public Health, 36(1), 68-72.

Honarmand, H., Hartskreel, R., Eshraghi, S., Khoramizadeh, M., \& Ghanaei, F. (2005). Study on the prevalence of Leptospirosis in Guilan Province, Iran. Paper presented at the 4th Scientific Meeting of the International Leptospirosis Society.

Joshi, Y. P., Kim, E.-H., \& Cheong, H.-K. (2017). The influence of climatic factors on the development of hemorrhagic fever with renal syndrome and leptospirosis during the peak season in Korea: an ecologic study. BMC infectious diseases, 17(1), 406. https://doi.org/10.1186/s12879-017-2506-6

Katz, A. R., Buchholz, A. E., Hinson, K., Park, S. Y., \& Effler, P. V. (2011). Leptospirosis in Hawaii, USA, 19992008. Emerging Infectious Diseases, 17(2), 221-226. https://doi.org/10.3201/eid1702.101109

Koe, S.-L. L., Tan, K. T., \& Tan, T. C. (2014). Leptospirosis in pregnancy with pathological fetal cardiotocography changes. Singapore Medical Journal, 55(2), e20-e24. https://doi.org/10.11622/smedj.2013194

Lehmann, J. S., Matthias, M. A., Vinetz, J. M., \& Fouts, D. E. (2014). Leptospiral Pathogenomics. Pathogens, 3(2), 280-308. https://doi.org/10.3390/pathogens3020280 
Mansour-Ghanaei, F., Sarshad, A., Fallah, M.-S., Pourhabibi, A., Pourhabibi, K., \& Yousefi-Mashhoor, M. (2005). Leptospirosis in Guilan, a northern province of Iran: assessment of the clinical presentation of 74 cases. Medical science monitor, 11(5), CR219-CR223.

Mehrotra, P., Ramakrishnan, G., Dhandapani, G., Srinivasan, N., \& Madanan, M. G. (2017). Comparison of Leptospira interrogans and Leptospira biflexa genomes: analysis of potential leptospiral-host interactions. Molecular bioSystems, 13(5), 883-891. https://doi.org/10.1039/c6mb00856a

Organization, W. H. (2010). Human leptospirosis: guidance for diagnosis, surveillance and control. 2003. World Health Organization: Geneva, Switzerland.

Palihawadana, P., Amarasekera, J., Ginige, S., Gamage, D., Jayasekera, S., \& Dayananda, M. (2014). The climatic factors associated with incidence of Leptospirosis in Sri Lanka. Journal of the College of Community Physicians of Sri Lanka, 19(2).

Sehgal, S. (2006). Epidemiological patterns of leptospirosis. Indian journal of medical microbiology, 24(4), 310.

Tasseva, E., Christova, I., Gladnishka, T., Trifonova, I., \& Ivanova, V. (2012). Leptospirosis in humans in Bulgaria-a new challenge for medicine or problem solved. Retrospective analysis of infection for 2010-2011. Science Infectology/Parasitology, 1, 22-29.

Yitzhaki, S., Barnea, A., Keysary, A., \& Zahavy, E. (2004). New Approach for Serological Testing for Leptospirosis by Using Detection of Leptospira Agglutination by Flow Cytometry Light Scatter Analysis. Journal of Clinical Microbiology, 42(4), 1680-1685. https://doi.org/10.1128/JCM.42.4.1680-1685.2004

Zakeri, S., Sepahian, N., Afsharpad, M., Esfandiari, B., Ziapour, P., \& Djadid, N. D. (2010). Molecular epidemiology of leptospirosis in northern Iran by nested polymerase chain reaction/restriction fragment length polymorphism and sequencing methods. Am J Trop Med Hyg, 82(5), 899-903. https://doi.org/10.4269/ajtmh.2010.09-0721

\section{Copyrights}

Copyright for this article is retained by the author(s), with first publication rights granted to the journal.

This is an open-access article distributed under the terms and conditions of the Creative Commons Attribution license (http://creativecommons.org/licenses/by/4.0/). 